\title{
Where's the evidence?-Making the case for public health
}

Tackling health inequalities has become a cornerstone of British government policy. From the corridors of Whitehall to the local board meetings of primary care groups and trusts, the imperative to reduce the health gap between rich and poor is ubiquitous. Yet when action is proposed to turn the rhetoric into reality, particularly when significant investments are required, the cry of "not enough evidence" is invariably heard in opposition.

Over the past decade, evidence-based practice has, quite properly, become a mantra of modern medicine. With the National Institute for Clinical Excellence and the Commission for Health Improvement now in place, medical practice in the NHS cannot get away with being anything other than evidence-based. The problem, though, is that the evidence most readily accepted within the health system comes from scientific methodologies like randomised control trials, which may not be the most appropriate forms of research into health improvement activities like community development, smoking cessation and rehabilitation. The growing understanding that health should be seen holistically — as being more about general wellbeing than the absence of disease-has not yet been equipped with evidence that policymakers and practitioners can respect.

Throughout the history of the NHS, there has been a tension between the imperative to treat disease in the individual and the need to promote general good health in the population. The two activities have invariably clashed over access to resources and demand on health workers' time. On some occasions, they have come into a more direct conflict, for example during the current arguments about the safety of the MMR vaccine and the value of fluoridation in tap water. With the NHS National Plan recently published, those tensions look set to continue. On the one hand, ministers have promoted health improvement activities and made reducing inequalities a central objective for the NHS, while on the other hand they have set ambitious targets for increasing elective surgery activity rates, which include asking family doctors to carry out some minor operations. The labour of health professionals is the most scarce resource in our health system, and it looks set to be the focus of fierce competition for some time to come.

How, then, can health improvement activities get a fair share of that time? While there is no simple answer to that question, it is surely important that sufficient evidence of its effectiveness is collected and disseminated among those who decide how health workers' time is divided up, from national policymakers at the top to local GPs at the "coalface". But for that to happen, there needs first to be a meeting of the minds between all those organisations and individuals whose involvement could be important for reducing the health gap.

For anyone promoting health improvement activities locally, building a robust and purposeful partnership can be one of the toughest jobs of all. Persuading NHS practitioners, local government officials, housing agencies, criminal justice organisations and private businesses, as well as communities themselves, to work together on a common project with a shared goal can feel next to impossible. When building such alliances, however, developing a shared understanding from the very start can be among the most crucial activities undertaken. In public health, for example, it is vital that all the different perspectives on a locality's problems are collected and examined. It may then be possible to identify some initial goals for health improvement in that area around which the partners involved can work collectively. At that point, it may then be possible to examine what activities could be undertaken to move towards those goals, and how progress-or lack of it - can be measured.

If a genuinely holistic approach is being taken, it is probable that several activities will be happening simultaneously to tackle any given problem, and that more than one measure of success would be appropriate. Collecting evidence to prove what works and what does not may also take a varied approach that mixes different methodologies and creates some new ones. In small localities, for example, measuring death rates from coronary heart disease over quite short periods of time is unlikely to be statistically valid. But looking at rates of smoking cessation or dietary changes may be more appropriate as signifiers of improvement in health. If a target of health improvement is to reduce the burden of mental ill health, measures might include a reduction in the number of prescriptions of antidepressant medication (bearing in mind that increasing numbers may be a positive sign at the start of a campaign that raises awareness of the problem), the number of people with mental illnesses finding work or educational opportunities (as a means of reducing isolation) or using focus groups to discuss whether interventions to tackle the causes of stress and anxiety (for example improved community safety) have worked.

Initiatives of this kind will need considerable support beyond the local level. At the national level, the new Health Development Agency, and the forthcoming Healthy Communities Collaborative, can give more weight to the evidence collected from successful initiatives and produce guidelines for other localities. The NHS regional offices, in conjunction with the new regional development agencies and government offices, can do much to promote partnerships and show leadership by example. Support at the national level may also need to extend to alterations in funding and accountability arrangements that encourage partnership working and that judge the effectiveness of public services by evidence of health improvement in their localities. Before that happens, though, the ministries in Whitehall will need to begin to talk the same language as one another and to develop their own shared understandings in the same manner as their local offshoots.

We are still some way from building a body of evidence about what works in the health improvement field. It is also possible we may never have one. Each locality, and each community, will have its own ideas about what prevents them from enjoying better health. To that end, one of the most important reforms promised in the National Plan for the NHS is an imperative to listen to local people much more than previously. An NHS that was more accountable to the communities it serves - not just to individual "consumers" but to citizens collectively-would be far more capable of putting time and money into health improvement ventures. Freeing NHS managers from the endless pursuit of centrally imposed targets could help them to respond more freely to local needs and to experiment with new approaches to community health, on the understanding that some of them will not meet the targets set for them.

What this all adds up to is the need for a culture of experiment, of listening, and of sharing. There are signs that things are moving in the right direction. Changes in the way that health professionals are being trained should 
help future doctors, nurses and others to think more holistically and to share more knowledge. Health Improvement Programmes, and the proposed community plans to be drawn up by local authorities, may provide good vehicles for initiatives that can break new ground. The uncertainty surrounds whether all the developments that are happening will fit together, and to what extent communities themselves will hold any real power, or responsibility, in them.

For public health to receive the resources it desperately needs, then, a combination of national and local action is required. In each case, there will need to be shifts towards effective partnership working between relevant agencies, and towards giving communities themselves a much bigger say in how resources are allocated to health improvement. We need, in other words, to be able to build the evidence base but at the same time change the way we look at evidence and to work much more closely around the views and experiences of the people for whom health improvement activities are designed.

RABBI JULIA NEUBERGER

The King's Fund, 11-13 Cavendish Square, London W1G OAN, UK 